\title{
40. Possible Interaction of Interstellar Particles With the Solar and Terrestrial Environment
}

\author{
J. Mayo Greenberg \\ State University of New York at Albany and Dudley Observatory \\ Albany, New York
}

\begin{abstract}
The possibility for detection of interstellar particles in the Earth's environment is considered on the basis of the passage of the solar system through the interstellar medium. Among the forces which inhibit interstellar particle penetration, the deflection by the solar magnetic field and the repulsive force due to the radiation from the Sun are by far the most important.
\end{abstract}

$l^{2}$

T IS VARIOUSLY ESTIMATED that the number density of small particles in the solar system at one AU is of the order of $10^{-12} \mathrm{~cm}^{-3}$. If we use an average interstellar extinction per unit distance in the solar neighborhood as $1 \mathrm{mag} / \mathrm{kpc}$ we obtain an average number density for $0.1 \mu$ radius interstellar particles of $N_{I P} \cong 10^{-12} \mathrm{~cm}^{-3}$ which is the same order of magnitude.

While it is true that the interstellar particles are generally smaller than the interplanetary particles, nevertheless the small particle component of the interplanetary space which contributes particularly to the polarization of the zodiacal light is suggestively similar in typical size (Giese and Dziembowski, 1967, Aller et al., 1967). It is therefore not unreasonable to examine further the question of the interstellar component of the interplanetary particle distribution (Greenberg, $1969,1970)$. We must consider first the chance for passage of the Sun through an interstellar cloud and further we must consider the effects of repulsive forces exerted by the solar system on the incoming interstellar particles.

\section{PASSAGE OF THE SUN THROUGH AN INTERSTELLAR CLOUD}

The space between the stars appears to consist mainly of cool neutral hydrogen clouds imbedded in a hot neutral hydrogen medium of quite low density. The cool clouds vary in size typically between $1 \mathrm{pc}$ and $10 \mathrm{pc}$ in radius. We picture a spherical shape for illustrative purposes only. The larger clouds may have a hydrogen number density of $N_{H}=10 \mathrm{~cm}^{-3}$ and the smaller ones may have a density of $N_{H}=10^{3} \mathrm{~cm}^{-3}$ or higher. Neither the distribution of cloud size nor the appropriate densities is firmly established. For our purposes it will be useful to make some crude assumptions to get order of magnitude estimations of the probability that the Sun may be passing through some cloud. It should be noted that with the hydrogen densities chosen above the respective particle densities in small and large clouds would be the order of $4000 \times 10^{-12} \mathrm{~cm}^{-3}$ and $40 \times 10^{-12}$ $\mathrm{cm}^{-3}$ which are larger than the number density of small zodiacal light particles.

If all the clouds are of $10 \mathrm{pc}$ radius, the number density of such clouds would be $6 \times 10^{3} \mathrm{kpc}^{-3}$ and if all are $1 \mathrm{pc}$ radius clouds the number density would be $6 \times 10^{4} \mathrm{kpc}^{-3}$. Using random cloud velocities of $10 \mathrm{~km} / \mathrm{sec}$ ( $10 \mathrm{pc}$ in one million years) we see that the time of passage through the large and small clouds are $10^{6}$ and $10^{5}$ years respectively. Based on the cloud number densities the time spent between the clouds is, for the former, $5 \times 10^{6} \mathrm{yrs}$. and, for the latter, $2.5 \times 10^{6}$ yrs. Thus the fraction of time spent inside a cloud 
is bracketed between $1 / 5$ and $1 / 25$. Neither of these probabilities-certainly not the former-is sufficiently small to exclude the chance that some of the interplanetary particles are of interstellar origin. It should be pointed out on the other hand that there is apparently no convincing evidence that we are now passing through a cloud.

\section{DUST REPELLING MECHANISMS}

Three obvious mechanisms for repelling the interstellar dust from entering the solar system are magnetic fields, the solar wind and solar radiation pressure. The first is applicable only if the dust particles are charged. The effects of the second are stronger if the dust particles are charged but exists even when the grains are uncharged.

\section{Solar Wind}

Let us estimate the distance over which an interstellar grain will penetrate the solar system under the assumption that it meets the solar wind head-on. We assume that the solar wind consists of 3 protons $\mathrm{cm}^{-3}$ moving with a speed of $400 \mathrm{~km}$ $\sec ^{-1}$. The penetration distance $d$ is obtained by requiring that the total energy imparted to the grain by the colliding protons equal the initial grain kinetic energy. We may write this condition approximately as $1 / 2 m_{0} V^{2}=m_{H} v_{H}^{2} n_{H} \pi a^{2} d$. Using grain density $s=1$ and radius $a=10^{-5} \mathrm{~cm}$ we find that $d \approx 50 \mathrm{AU}$ if the speed of passage of the solar system through the cloud is $10 \mathrm{~km} / \mathrm{sec}$. This is so large insofar as the solar wind is concerned that the interstellar grains should pass through unaffected. In view of the fact that we have assumed a constant solar wind intensity throughout the solar system in this simplified calculation, we have greatly overestimated the solar wind effect. Even taking into account the fact that a charged grain would have a greater collision cross section, the average rate of momentum transfer for the distant collisions is not sufficient to change our conclusions.

\section{Magnetic Field}

As an illustration we consider the effect on a "typical sized" $a=0.1 \mu$ grain. Larger grains would be less deflected than smaller grains so the criticality derived is an upper limit insofar as larger grains are concerned.

Rather than calculating the radius of curvature of a typical entering grain we calculate its deflection. The calculation is simplified by assuming the net effect will not be large. The final result will indicate the consistency of this assumption.

Consider the worst possible case that the grain enters normal to the magnetic field in the solar system. The field will be represented by its transverse component $B_{t}$, (Ness, 1968) having a field strength of the order of $6 \times 10^{-5}$ gauss at $1 \mathrm{AU}$.

The force on the grains is $q V B$ and the acceleration is $a_{\perp}=q V B_{t} / m_{g}$ where $B_{t}=B_{0}(1 \mathrm{AU} / R)$ and $B_{0}=6 \times 10^{-5}$ gauss. The deflection may be calculated simply by assuming a very large radius of curvature. We get the lateral deflection as

$$
\Delta=\int_{0}^{t} a_{\perp} t d t
$$

We let $t=x / V$ where $x$ is measured from some outer point in the solar system. We shall choose this outer point as $R_{0}=10 \mathrm{AU}$. Then:

$$
\Delta=\frac{q V B_{0}(1 \mathrm{AU})^{2}}{m_{0} V^{2}} \int_{R_{1}}^{R_{0}} \frac{\left(R_{0}-R\right) d R}{R}
$$

where $R_{1}$ is chosen as the distance from the Sun where the deflection is defined. If we let $R_{1}=1 \mathrm{AU}$ and let the charge on the grain be such as to give it a one volt potential we get $\Delta \approx 10^{3} \mathrm{AU}$ for $V=20 \mathrm{~km} / \mathrm{s}$ ! This is enormous. It is only for grains with potentials $\leq 10^{-3}$ volts that the angle of deflection becomes moderate. For $a=10^{-5} \mathrm{~cm}$ this implies less than one electron charge. We note that, for fixed potential, $\Delta \sim a^{-2}$ so that interstellar grains in the one micron size range might sweep through.

\section{Radiation Pressure}

The criterion we use here is whether the net repulsive force is such that an incoming giain at $10 \mathrm{~km} / \mathrm{sec}$ turns around at $1 \mathrm{AU}$. We therefore equate the initial kinetic and final potential energy where the latter is defined in terms of an effective repulsive gravitational constant $\epsilon G$. Thus $\epsilon \equiv$ $\left(1 / 2 V^{2}\right) /\left(G M_{0} / R_{1}\right)$. This gives $\epsilon \approx 0.05$, i.e., the radiation force need be only slightly greater than 
the gravitational force. For a $6000 \mathrm{~K}$ blackbody the radiation force on a $0.1 \mu$ dirty ice grain is $\approx 10^{-10}$ dynes. The gravitational force is equivalently $4.3 \times 10^{-10}$ dynes. Thus a $0.1 \mu$ ice grain should penetrate. It can be shown that somewhat smaller grains will also penetrate. However it is clear that the dominant factor in preventing the penetration of dust particles from interstellar space is the radiation of the Sun.

\section{ACKNOWLEDGMENT}

Work supported in part by NASA Grant No. NGR33-011-043.

\section{REFERENCES}

Aller, L. H., Duffner, G., Dworetsky, M., Gudehus, D., Kilston, S., Leckrone, D., Montgomery, J., Outver, J., And Zimmerman, E., 1967. Some models of the zodiacal cloud, The Zodiacal Light and the Interplanetary Medium, edited by J. L. Weinberg, NASA SP-150, Supt. of Documents, U.S. Govt. Printing Office, Washington, 243-256.

Giese, R. H., AND Dztembowski, C. V., 1967. On optical models approximating observations of the zodiacal light outside the ecliptic, The Zodiacal Light and the Interplanetary Medium, edited by J. L. Weinberg, NASA SP-150, Supt. of Documents, U.S. Govt. Printing Office, Washington, 271-276.

Greenberg, J. M., 1969. A possible inter-relation between interstellar and interplanetary cosmic dust, Space Research $I X, 111-115$.

GreEnBerg, J. M., 1970. Models of the zodiacal light, Space Research X, 225-232.

Ness, N. F., 1968. Observed properties of the interplanetary plasma, Ann. Rev. Astron. Astrophys., $6,79-114$. 